\title{
LINEAR STATE REGULATORS AND INTEGRAL EQUATIONS NOT REDUCIBLE TO DIFFERENTIAL EQUATIONS
}

\author{
Donald R. Smith \\ Dedicated to the memory of Charles G. Lange.
}

\begin{abstract}
Examples are given on an application in optimal control theory leading to a Fredholm integral equation that is not reducible by standard procedures to an equivalent differential equation problem. The stipulated optimization problem involves a Volterra state constraint that is itself not reducible to a differential equation. The stated class of problems includes singularly perturbed Fredholm problems of the type considered in $[1,2]$. Such problems cannot be handled by standard techniques in the singular perturbation theory of differential equations.
\end{abstract}

\section{Introduction}

Vector (and scalar) singularly perturbed Fredholm integral equations of the form

$$
\int_{0}^{1} K(t, s) w(s) d s=h(t, \epsilon)+\epsilon w(t), \quad 0 \leq t \leq 1
$$

with kernels $K$ possessing suitable jump discontinuities are considered in $[1,2]$ for small positive values of $\epsilon$ subject to suitable assumptions. The leading-order outer solution generally does not satisfy the reduced integral equation obtained by setting $\epsilon=0$ in (1.1), and the solution of (1.1) generally exhibits large amplitude boundary layer behavior at one or both endpoints. The perturbation technique of $[1,2]$ is based on an efficient asymptotic splitting of the integral equation which reveals the asymptotic structure of solutions. The resulting asymptotic expansion is used in [1] to prove existence and uniqueness of solutions of boundary-layer type for the considered class of equations even though the reduced equation may have no solution or many solutions depending on the kernel $K$ and the forcing function $h$.

It was noted in $[1,2]$ that linear singularly perturbed two-point boundary-value problems for differential equations can be packaged, via a suitable Green function, in the form of an appropriate integral equation such as (1.1), so that such differential equation boundary-value problems lead to examples of (1.1). The asymptotic procedure of $[1,2]$ deals directly with the integral equation (1.1) and makes no use of any possible equivalent differential equation problem. When the asymptotic procedure of $[1,2]$ is applied to an integral equation that is equivalent to an associated differential equation problem, the results are equivalent to the known results obtained by standard techniques in singular perturbation theory for differential equations (as discussed for example in [4]).

An optimal control problem is considered here that provides an integral equation (1.1) which generally cannot be reduced to an associated differential equation problem.

Received November 16, 1993, revised October 5, 1994. 
For such problems the standard differential equation methods are not applicable, but these problems can be studied with the perturbation technique of $[1,2]$.

\section{A perturbed linear state regulator}

Consider the problem of minimizing the scalar functional

$$
\mathcal{C}[u]:=\frac{1}{2} \int_{0}^{1}\left[\beta(t)^{2} x(t)^{2}+u(t)^{2}\right] d t
$$

subject to the scalar constraint

$$
\epsilon x(t)=f(t, \epsilon)+\int_{0}^{t} \alpha(t, s) u(s) d s \quad \text { for } 0 \leq t \leq 1 .
$$

The related vector case can be handled directly by the same methods but, for simplicity of exposition, only the scalar case is considered here. The scalar $x(t) \equiv x(t ; u)$ is the state function while $u(t)$ is a scalar control function. The constraint (2.2) determines the state function uniquely in terms of any chosen control function $u$, and one seeks a particular control function over the fixed time horizon $0 \leq t \leq 1$ that will minimize the cost functional (2.1). The data functions $\alpha, \beta$. and $f$ are assumed to be continuous, with $\beta$ assumed to be everywhere positive while $\alpha$ is nonzero and may (by continuity) be assumed to be everywhere positive (otherwise replace $\alpha$ by $-\alpha$ ),

$$
\alpha(t, s)>0 \text { for } 0 \leq t, s \leq 1, \quad \text { and } \beta(t)>0 \text { for } 0 \leq t \leq 1 .
$$

The extremal problem (2.1)-(2.2) may be discussed within the framework of the Hamiltonian formulation of the calculus of variations but we shall instead follow the classical variational approach here, according to which an optimizing control function must satisfy Euler's necessary condition

$$
\delta \mathcal{C}[u ; \Delta u]=0 \text { for all functions } \Delta u .
$$

A routine calculation from (2.1)-(2.2) yields for the variation (cf. [3])

$$
\delta \mathcal{C}[u ; \Delta u]=\int_{0}^{1}\left[u(t)+\frac{1}{\epsilon} \int_{t}^{1} \beta(s)^{2} \alpha(s, t) x(s) d s\right] \Delta u(t) d t,
$$

and then (2.4)-(2.5) and the arbitrariness of $\measuredangle u$ lead to the Euler equation

$$
\epsilon u(t)=-\int_{t}^{1} \beta(s)^{2} c x(s, t) x(s) d s
$$

which must be satisfied along with the given constraint equation (2.2). In particular it follows that the optimizing functions $x, u$ must satisfy the natural boundary conditions

$$
x(0)=\frac{1}{\epsilon} f(0, \epsilon) \quad \text { and } \quad u(1)=0 .
$$

The system $(2.2),(2.6)$ can be written as the vector integral equation

$$
\epsilon\left(\begin{array}{l}
x(t) \\
u(t)
\end{array}\right)+\left(\begin{array}{c}
-f(t, \epsilon) \\
0
\end{array}\right)=\int_{1}^{1} K(t, s)\left(\begin{array}{l}
x(s) \\
u(s)
\end{array}\right) d s
$$


with kernel

$$
K(t, s)= \begin{cases}K^{-}(t, s)=\left(\begin{array}{cc}
0 & \alpha(t, s) \\
0 & 0
\end{array}\right) & \text { for } s<t \\
K^{+}(t, s)=\left(\begin{array}{cc}
0 & 0 \\
-\beta(s)^{2} \alpha(s, t) & 0
\end{array}\right) & \text { for } s>t .\end{cases}
$$

This kernel possesses the jump

$$
J[K](t):=K^{-}\left(t, t^{-}\right)-K^{+}\left(t, t^{+}\right)=\left(\begin{array}{cc}
0 & \alpha(t, t) \\
\beta(t)^{2} \alpha(t, t) & 0
\end{array}\right),
$$

and the eigenvalues $\lambda$ of this jump matrix (2.10) are

$$
\lambda= \pm \alpha(t, t) \beta(t) \quad \text { for } \quad 0 \leq t \leq 1 .
$$

Hence (2.8) is of the form of equation (1.1) with

$$
w=\left(\begin{array}{l}
x \\
u
\end{array}\right), \quad h(t, \epsilon)=\left(\begin{array}{c}
-f(t, \epsilon) \\
0
\end{array}\right)
$$

and with kernel $K$ given by (2.9).

A key assumption on the equation (1.1) in [1] requires that all eigenvalues of the jump matrix must have nonzero real parts (cf. [1, (5.1)]), and one sees with (2.3) and (2.11) that problem (2.8)-(2.11) satisfies this condition and is of the type considered in $[1]$.

\section{Examples}

In certain cases, the Fredholm equation (2.8)-(2.9) can be reduced to a boundary-value problem for an associated differential equation, as occurs for example if the function $\alpha(t, s)$ is independent of $t$,

$$
\frac{\partial \alpha(t, s)}{\partial t} \equiv 0, \quad \alpha(t, s)=\alpha(s) \quad \text { for } \quad 0 \leq t, s \leq 1
$$

for some continuously differentiable function $\alpha(s)$. In this case, one finds upon differentiation that $(2.8)$ is equivalent to the differential equation

$$
\epsilon \frac{d}{d t}\left(\begin{array}{l}
x \\
u
\end{array}\right)=\left(\begin{array}{cc}
0 & \alpha(t) \\
\beta(t)^{2} \alpha(t) & \epsilon \alpha^{\prime}(t) / \alpha(t)
\end{array}\right)\left(\begin{array}{l}
x \\
u
\end{array}\right)+\left(\begin{array}{c}
f^{\prime}(t, \epsilon) \\
0
\end{array}\right) \quad \text { for } 0 \leq t \leq 1
$$

along with the boundary conditions (2.7). Standard techniques from the theory of singularly perturbed differential equations can be used in the study of (2.7), (3.2).

To illustrate the flavor of such results consider the trivial case of constant data

$$
\alpha(t, s) \equiv \alpha=\text { const. and } \beta(t) \equiv \beta=\text { const. }
$$

in which case the perturbed linear state regulator $(2.1),(2.2)$ or the equivalent boundary-value problem (2.7), (3.2) can be easily solved in closed form. For example, if the forcing function is

$$
f(t, \epsilon)=e^{\alpha \gamma t} \quad \text { for } \quad t \geq 0
$$

then one finds 


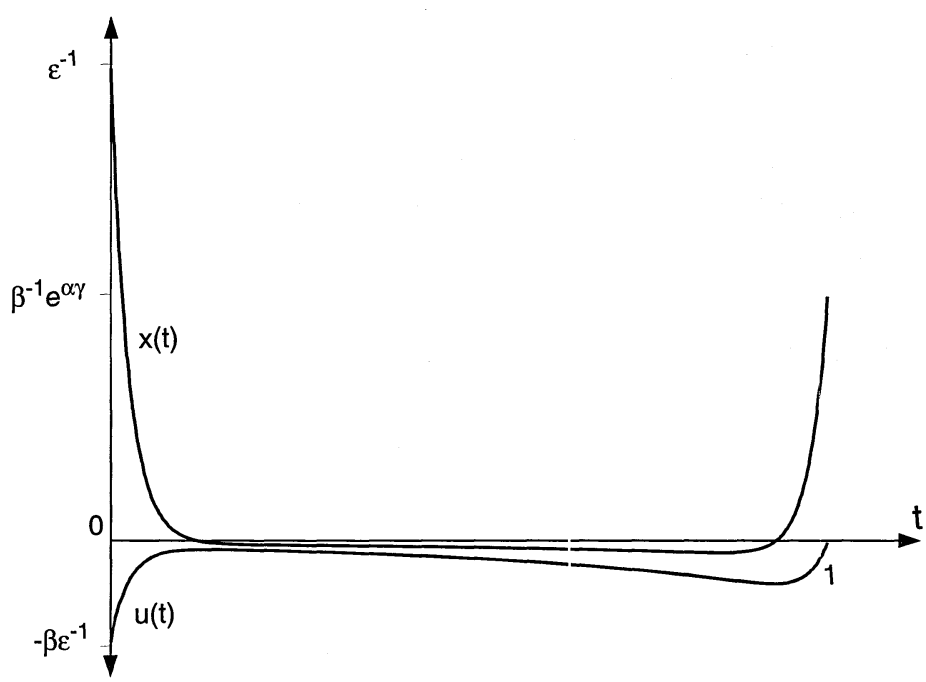

Figure 1

$$
\begin{aligned}
x(t)= & {\left[\frac{\beta^{2}-\epsilon^{2} \gamma^{2}+\epsilon^{2} \gamma}{\epsilon\left(\beta^{2}-\epsilon^{2} \gamma^{2}\right)}\right] \frac{e^{-\alpha \beta t / \epsilon}+e^{-\alpha \beta(2-t) / \epsilon}}{1+e^{-\alpha \alpha \beta / \epsilon}} } \\
& -\left(\frac{\epsilon \gamma}{\beta^{2}-\epsilon^{2} \gamma^{2}}\right) e^{\alpha \gamma t}+\left[\frac{\beta e^{\alpha \gamma}}{\beta^{2}-\epsilon^{2} \gamma^{2}}\right] \frac{e^{-\alpha \beta(1-t) / \epsilon}-e^{-\alpha \beta(1+t) / \epsilon}}{1+e^{-2 \alpha \beta / \epsilon}} \\
u(t)= & {\left[\frac{\beta\left(\beta^{2}-\epsilon^{2} \gamma^{2}+\epsilon^{2} \gamma\right)}{\epsilon\left(\beta^{2}-\epsilon^{2} \gamma^{2}\right)}\right] \frac{-e^{-\alpha \beta t / \epsilon}+e^{-\alpha \beta(2-t) / \epsilon}}{1+e^{-2 \alpha \beta / \epsilon}} } \\
& -\left(\frac{\beta^{2}}{\beta^{2}-\epsilon^{2} \gamma^{2}}\right) e^{\alpha \gamma t}+\left[\frac{\beta^{2} e^{\alpha-\gamma}}{\beta^{2}-\epsilon^{2}}\right] \frac{e^{-\alpha \beta(1-t) / \epsilon}-e^{-\alpha \beta(1+t) / \epsilon}}{1+e^{-2 \alpha \beta / \epsilon}}
\end{aligned}
$$

from which

$$
\begin{aligned}
& x(t)=\frac{1}{\epsilon} e^{-\alpha \beta t / \epsilon}\left[1+\epsilon^{2} \frac{\gamma-\gamma^{2}+\beta^{2} \gamma^{2}}{\beta^{2}}\right]-\frac{\epsilon \gamma}{\beta^{2}} e^{\alpha \gamma t}+\frac{e^{\alpha \gamma}}{\beta} e^{-\alpha \beta(1-t) / \epsilon}+O\left(\epsilon^{2}\right) \\
& u(t)=-\frac{\beta^{2}+\epsilon^{2} \gamma}{\epsilon \beta} e^{-\alpha \beta t / \epsilon}-e^{\alpha \gamma t}+e^{\alpha \gamma} e^{-\alpha \beta(1-t) / \epsilon}+O\left(\epsilon^{2}\right)
\end{aligned}
$$

uniformly for $0 \leq t \leq 1, \epsilon \rightarrow 0$ as indicated in ct typical case in Figure 1 .

Unlike the situation for the elementary example (3.3), the Fredholm equation (2.8) cannot be reduced to a differential equation whenever the Volterra constraint (2.2) cannot be so reduced, as occurs for example in the case $\alpha(t, s)=(1+t+s)^{-1}$. This latter situation will generally occur for Volterra problems in which the past history of some quantity plays an important role. Indeed the origin of Volterra's work in integral equations is found in his earlier work in hereditary elasticity where, for example, the angle of twist $x(t)$ of a rod depends on the history of the twisting moment (or couple of torsion) $u(t)$ in the fashion (2.2). Such problems which involve the history of some relevant quantity also occur in viscoelasticity (cf. Section 5.7 of [5]) and in certain areas of mathematical physics where, for example, the electric or magnetic polarization 
depends on the history of the electromagnetic state of the matter (cf. Whittaker's biography of Volterra in [6]).

As an elementary example, consider a simple electric circuit with zero inductance and zero resistance containing only a capacitor with small hereditary capacitance $C$ given as

$$
C(t, s)=\frac{\epsilon}{\alpha(t, s)}
$$

for a small parameter $\epsilon$ which is a measure of the smallness of the capacitance, and where $\alpha=\alpha(t, s)$ is a positive order-unity scaled (reciprocal) capacitance which is defined by (3.7). Kirchhoff's voltage model and Coulomb's model of capacitance give (with initial time taken as $t=0$ )

$$
\epsilon E(t)=\int_{0}^{t} \alpha(t, s) i(s) d s
$$

for the voltage $E$ and current $i$. This is equation (2.2) with control function $u=i$ and state function $x=E$, and one then obtains a singularly perturbed Fredholm equation that cannot be reduced to a differential equation if one wishes to determine the current distribution $i(t)$ so as to minimize a cost functional of the form (2.1).

Applications also occur in areas of investment planning (cf. Section 3.7 of [3]) and production planning for manufacturing processes. For example, consider the case of a company that manufactures and sells some particular product subject to substantial spoilage, with sufficient long-term orders on hand so that the company can predict its future sales rate $\mathcal{S}=\mathcal{S}(t)$ with certainty over a fixed time horizon $0 \leq t \leq 1$. Based on the given sales rate $\mathcal{S}$ (and taking into account the spoilage) the company projects a desired inventory level $\mathcal{I}(t)$ and corresponding desired production level $\mathcal{P}(t)$. Assume however that the actual inventory level $I(t)$ is disturbed (perhaps by a malfunction at the manufacturing plant) away from its desired level $\mathcal{I}$ at time $t=0$ with $I(0)=I_{0} \neq \mathcal{I}(0)$, and the company then seeks a new actual production rate $P(t)$ that will guide the system back toward its desired state over a unit time interval so as to minimize a quadratic cost functional. If $\alpha(t, s) / \epsilon$ represents the (large) spoilage factor at time $t$ of inventory produced at time $s$, then one has a problem of the type (2.1)-(2.2) with (cf. [3, pp. 16-18, 42, 261-272])

$$
f(t, \epsilon)=-\epsilon \mathcal{I}(t)+\alpha(t, 0) I_{0}+\int_{0}^{t} \alpha(t, s)[\mathcal{P}(s)-\mathcal{S}(s)] d s
$$

In modelling possible seasonal and other effects, the spoilage factor $\alpha(t, s)$ must be considered to be a given but essentially arbitrary function taken from some appropriate class such as the class of all piecewise smooth functions for $0 \leq t, s \leq 1$. The state function $x=I-\mathcal{I}$ is the difference between the (sought) actual and (given) desired inventory levels and similarly the control function $u=P-\mathcal{P}$ is the difference between the (sought) actual and (given) desired production levels. The resulting singularly perturbed vector Fredholm equation (2.8), which cannot generally be reduced to a differential equation, can be handled directly by the approach of $[1,2]$.

One can also consider related vector linear regulator problems with vector state functions and vector control functions and with more complicated cost functionals. 


\section{References}

1. C. G. Lange and D. R. Smith, Singular perturbation analysis of integral equations, Stud. Appl. Math. 79 (1988), 1-63.

2. —_ Singular perturbation analysis of integral equations: part II, Stud. Appl. Math. 90 (1993), 1-74.

3. D. R. Smith, Variational Methods in Optimization, Prentice-Hall, Englewood Cliffs, N. J., 1974.

4. _ Singular Perturbation Theory, Cambridge University Press, Cambridge, 1985.

5. _ An Introduction to Continuum Mechanics, Kluwer Academic Publishers, Dordrecht, Boston, London, 1993.

6. V. Volterra, Theory of Functions and of Integro-Differential Equations, Dover Edition, 1959.

Department of Mathematics, University of Califcirnia at San Diego, la Jolla, CA 92093, U.S.A. 